\title{
OBTENÇÃO DE ANTOCIANINA EM PÓ A PARTIR DO RESÍDUO AGROINDUSTRIAL DA UVA ISABEL
}

\section{R. F. CONTINI, L. P. L. CILLI, C. M. P. YOSHIDA, M.B. BRAGA²,} A. C. VENTURINI ${ }^{1}$,

\author{
${ }^{1}$ Universidade Federal de São Paulo, Instituto de Ciências Ambientais, Químicas e \\ Farmacêuticas (ICAQF) \\ E-mail para contato: anna.venturini@unifesp.br \\ ${ }^{2}$ Universidade Federal de São Paulo, Departamento de Ciências Exatas e da Terra \\ E-mail para contato: matheus19braga@yahoo.com.br
}

\section{RESUMO}

Os resíduos agroindustriais de uva possuem compostos de alto valor nutricional e agregado como as fibras e compostos fenólicos, destacando as antocianinas que podem atuar como antioxidantes, porém são compostos de baixa estabilidade. A microencapsulação por spray dryer é uma alternativa para aumentar a estabilidade das antocianinas, visando a retenção deste composto. Assim, o objetivo do estudo foi determinar a concentração e a retenção das antocianinas proveniente da polpa de casca de uva-concentrado protéico do soro do leite em pó. Para a secagem por atomização as condições operacionais variáveis foram: temperatura do ar de secagem; vazão de alimentação da pasta; concentração de adjuvante. Observou-se que apenas o parâmetro temperatura do ar de secagem, apresentou efeito estatisticamente significativo em relação à concentração e retenção de antocianinas $(\mathrm{P} \leq 0,05) \mathrm{O}$ teor de antocianinas variou de 4,28 a 16,58 mg de cianidina-3-glucosídeo $100 \mathrm{~g}^{-1}$ de pó em base seca e a retenção entre $19,3 \%$ e 94,0 \%.

\section{INTRODUÇÃO}

Os resíduos da indústria de suco de uva representam cerca de $30 \%$ do volume total de uvas utilizadas para a produção de vinho (SELANI et al., 2012). Estes subprodutos, tais como sementes e cascas, são ricos em antocianinas, pigmentos solúveis em água, responsáveis por grande variedade de cores que variam do vermelho vivo ao violeta. Estes compostos podem atuar como antioxidantes e inibidores de enzimas envolvidas no estresse oxidativo, diabetes tipo 2, hipertensão e inflamação (LAVELLI et al., 2017).

As cascas de uva são fontes potenciais de fibra alimentar e compostos fenólicos, particularmente flavonóis, flavanóis, antocianinas e proantocianidinas (PEREZ-JIMENEZ et 


\section{Congresso Brasileiro de Engenharia Química \\ em Iniciação Científica \\ UFSCar - São Carlos - SP \\ 16 a 19 de Julho de 2017}

QUIMICA EM INICIACÃO CIENTÍFICA

al., 2008). Por este motivo há um grande interesse na possibilidade de converter este resíduo em ingrediente alimentar de alto valor agregado devido à sua capacidade de fornecer alegações de saúde ao produto final (GALANAKIS, 2015).

A secagem por atomização é uma boa alternativa para conservar a solução aquosa do extrato obtido a partir de resíduos de agroindustriais. Este processo facilita a embalagem, o armazenamento e a comercialização, além de proteger os compostos bioativos de reações químicas e bioquímicas e impedir o crescimento de microrganismos que podem degradar o extrato (SILVA et al., 2014).

O objetivo deste trabalho foi obter um pó contendo antocianinas a partir da polpa de casca de uva através da secagem por atomização utilizando concentrado proteico de soro de leite como agente adjuvante.

\section{MATERIAIS E MÉTODOS}

\subsection{Preparo da polpa}

O resíduo da industrialização do suco de uva (casca e caroço) utilizado nesse trabalho foi cedido pela empresa Produtos Alimentícios Superbom Indústria e Comércio Ltda. As cascas separadas manualmente dos caroços de uva Isabel (Vitis labrusca) foram homogeneizadas com água potável em uma proporção em massa de 1:4, e a polpa formada foi filtrada em tecido para a eliminação de potenciais resíduos sólidos. Antes da microencapsulação das antocianinas em spray dryer o adjuvante concentrado proteico de soro de leite (Lacprodan $80^{\circledR}$ ) foi adicionado à polpa de casca de uva nas porcentagens de 10,12,5 e 15\% (em massa).

A Tabela 1 mostra a matriz do planejamento experimental com repetição do ponto central. As condições operacionais fixas foram: vazão do ar de secagem, 3,0 $\mathrm{m}^{3} \mathrm{~min}^{-1}$; pressão do ar de atomização, 2,93.10 $\mathrm{Pa}$; diâmetro do bico atomizador, 1,0 mm. As condições operacionais variáveis foram: temperatura do ar de secagem $(\mathrm{T})$; vazão de alimentação da pasta (W); concentração de adjuvante (A). 


\section{Congresso Brasileiro de Engenharia Química \\ em Iniciação Científica \\ UFSCar - São Carlos - SP \\ 16 a 19 de Julho de 2017}

Tabela 1- Matriz de planejamento experimental da atomização.

\begin{tabular}{|c|c|c|c|c|c|c|}
\hline Ensaio & $\mathbf{T}$ & $\mathbf{W}$ & $\mathbf{A}$ & $\mathbf{T}\left({ }^{\circ} \mathbf{C}\right)$ & $\begin{array}{c}\mathrm{W} \\
\left(\mathrm{g} \mathrm{\textrm {min } ^ { - 1 } )}\right)\end{array}$ & $\begin{array}{c}\text { A } \\
(\%)\end{array}$ \\
\hline 1 & -1 & +1 & +1 & 160 & 10 & 15 \\
\hline 2 & -1 & +1 & -1 & 160 & 10 & 10 \\
\hline 3 & -1 & -1 & +1 & 160 & 5 & 15 \\
\hline 4 & -1 & -1 & -1 & 160 & 5 & 10 \\
\hline 5 & +1 & +1 & +1 & 190 & 10 & 15 \\
\hline 6 & +1 & +1 & -1 & 190 & 10 & 10 \\
\hline 7 & +1 & -1 & +1 & 190 & 5 & 15 \\
\hline 8 & +1 & -1 & -1 & 190 & 5 & 10 \\
\hline 9 & 0 & 0 & 0 & 175 & 7,5 & 12,5 \\
\hline 10 & 0 & 0 & 0 & 175 & 7,5 & 12,5 \\
\hline 11 & 0 & 0 & 0 & 175 & 7,5 & 12,5 \\
\hline
\end{tabular}

Condições operacionais fixas ( $T, W$ e $A)$ e variáveis $\left(T=\right.$ temperatura de secagem em ${ }^{\circ} \mathrm{C}, \mathrm{W}=$ vazão de entrada de pasta em g. $\mathrm{min}^{-1} \mathrm{e} A=$ concentração de adjuvante na pasta em \%, em massa).

\subsection{Determinação da concentração de antocianinas totais no pó}

A concentração de antocianinas totais $\left(\mathrm{C}_{\text {ant }}\right)$ foi determinada pelo método de espectrofotometria diferencial de $\mathrm{pH}$ desenvolvido para sucos de frutas, bebidas, corantes naturais e vinhos descrito por LEE et al. (2005). A amostra microencapsulada (1 g) foi misturada com $20 \mathrm{~mL}$ de etanol:ácido clorídrico 1,5 N (85:15, em volume) e filtrada a vácuo. Uma alíquota de $1 \mathrm{~mL}$ das amostras foram dissolvidas em 0,025 M de solução tampão de cloridrato de potássio $(\mathrm{pH}=1,0)$ e em $0,4 \mathrm{M}$ de solução de acetato de sódio $(\mathrm{pH}=4,5)$, com o fator de diluição pré determinado em 10 ou 5 , de acordo com a coloração da amostra. A leitura da absorbância foi realizada em $510 \mathrm{~nm}$, considerando a absorbância máxima para cianidina-3glicosídeo e em $700 \mathrm{~nm}$ para descontar a turbidez da amostra. As leituras de absorbância (Abs) foram realizadas em espectrofotômetro (Evolution 60s, Thermo Scientific, China) e calculadas, usando-se a Equação 1:

$$
\operatorname{Abs}=\left[\left(\mathrm{Abs}_{510}-\mathrm{Abs}_{700}\right)_{\mathrm{pH} 1,0}\right]-\left[\left(\mathrm{Abs}_{510}-\mathrm{Abs}_{70}\right)_{\mathrm{pH}} 4,5\right](\text { Equação 1) }
$$

A concentração de antocianinas totais $\left(\mathrm{C}_{\mathrm{ant}}\right) \mathrm{em} \mathrm{mg} \mathrm{L}^{-1}$ foi calculada como cianidina-3glicosídeo a partir da Equação 2:

$$
\mathrm{C}_{\mathrm{ant}}\left(\frac{\mathrm{mg}}{\mathrm{L}}\right)=\frac{\text { AxPMxFDx } 10^{3}}{\varepsilon \times 1} \quad(\text { Equação } 2)
$$

onde $\mathrm{PM}=$ peso molecular $=449,2 \mathrm{~g} \cdot \mathrm{mol}^{-1}$ para cianidina-3-glicosídeo; $\mathrm{FD}=$ fator de diluição; $\varepsilon=$ absortividade molar $=26900$ mol.L $\mathrm{L}^{-1} ; 1=$ espessura da cubeta em $\mathrm{cm}$ e $10^{3}=$ fator para conversão de g para mg.

A retenção das antocianinas $\left(\mathrm{R}_{\mathrm{ant}}\right)$ foi determinada a partir da diferença de concentração destes pigmentos antes e após a passagem pelo secador. $\mathrm{O}$ teor de antocianinas foi expresso em (mg.100 g ${ }^{-1}$ de pó seco). 


\section{Congresso Brasileiro de Engenharia Química \\ em Iniciação Científica \\ UFSCar - São Carlos - SP \\ 16 a 19 de Julho de 2017}

QUÍMICA EM INICIACÃO CIENTÍFICA

\subsection{Análise estatística}

A análise estatística da matriz de planejamento foi realizada utilizando-se o software Statistica 13.0 (Tulsa, Estados Unidos), com um nível de confiança de 95\% ( $\leq \leq 0,05)$.

\section{RESULTADOS E DISCUSSÕES}

A concentração de antocianinas totais e a porcentagem de retenção das antocianinas obtidas para os 11 Ensaios de atomização estão apresentados na Tabela 1. O teor de antocianinas variou de 4,28 a 16,58 mg de cianidina-3-glucosídeo/100 g de pó em base seca.

Resultados similares foram obtidos por Silva, Constant, Figueiredo e Moura (2010) que secaram o extrato de casca de jabuticaba com maltodextrina em spray drier e obtiveram 11,8 mg de cianidina-3-glucosídeo.100 $\mathrm{g}^{-1}$ de pó em base seca. Valores superiores foram obtidos por SILVA et al. (2014) que atomizaram o extrato de casca de jabuticaba utilizando diferentes concentrações de maltodextrina e obtiveram de 7,0 a 21,6 mg de cianidina-3glucosido. $\mathrm{g}^{-1}$ de antocianina total em pó. Esses autores observaram que quanto maior a concentração do adjuvante na amostra, menor o teor de antocianina, e vice-versa. No entanto, nesse trabalho, a concentração do adjuvante Lacprodan $80^{\circledR}$ não teve um efeito diluente sobre a concentração do pigmento no pó.

Tabela 1- Concentração $\left(\mathrm{C}_{\mathrm{ant}}\right)$ e retenção das antocianinas $\left(\mathrm{R}_{\mathrm{ant}}\right)$ no pó.

\begin{tabular}{cccc}
\hline Ensaio & $\begin{array}{c}\mathrm{C}_{\text {ant }} \\
\left(m g .100 \mathrm{~g}^{-1} \text { b.s. }\right)\end{array}$ & $\mathrm{DM}^{*}$ & $\begin{array}{c}\mathrm{R}_{\text {ant }} \\
(\%)\end{array}$ \\
\hline 1 & 4,28 & 0,82 & 24,1 \\
2 & 6,87 & 0,00 & 26,0 \\
3 & 9,54 & 0,86 & 53,8 \\
4 & 4,88 & 1,33 & 19,3 \\
5 & 16,58 & 0,87 & 94,0 \\
6 & 14,50 & 0,85 & 54,8 \\
7 & 16,21 & 0,43 & 92,4 \\
8 & 11,73 & 0,43 & 45,1 \\
9 & 14,12 & 0,83 & 65,3 \\
10 & 9,04 & 0,21 & 41,6 \\
11 & 12,40 & 0,79 & 57,4 \\
\hline \multicolumn{4}{c}{}
\end{tabular}

*DM= Desvio médio

No processo de secagem por spray dryer a exposição de produto termossensível a altas temperaturas por curtos períodos de tempo, resulta em redução da perda de compostos termossensíveis (TONON, 2009).

Neste trabalho observou-se uma redução significativa na perda de antocianinas com o aumento da temperatura, embora a interação temperatura versus vazão não tenha apresentado 


\section{Congresso Brasileiro de Engenharia Química \\ em Iniciação Científica \\ UFSCar - São Carlos - SP

diferença significativa entre os 11 ensaios estudados.

Observou-se, nas faixas estudadas, que a vazão de entrada da pasta e concentração de adjuvante na polpa não foram estatisticamente significativos para a retenção de antocianinas no pó, para um nível de confiança de $95 \%$.

Analisando a Figura 1, observa-se que a temperatura teve efeito positivo na concentração de antocianinas no pó produzido, portanto, a melhor condição para a produção de pó, caso o parâmetro de maior interesse seja a concentração de antocianinas, é na maior temperatura, observada nos ensaios 5 e 7 da matriz de planejamento experimental.

Figura 1: Influência das condições operacionais na concentração de antocianinas do produto: gráfico de pareto $(\mathrm{P} \leq 0,05))$.

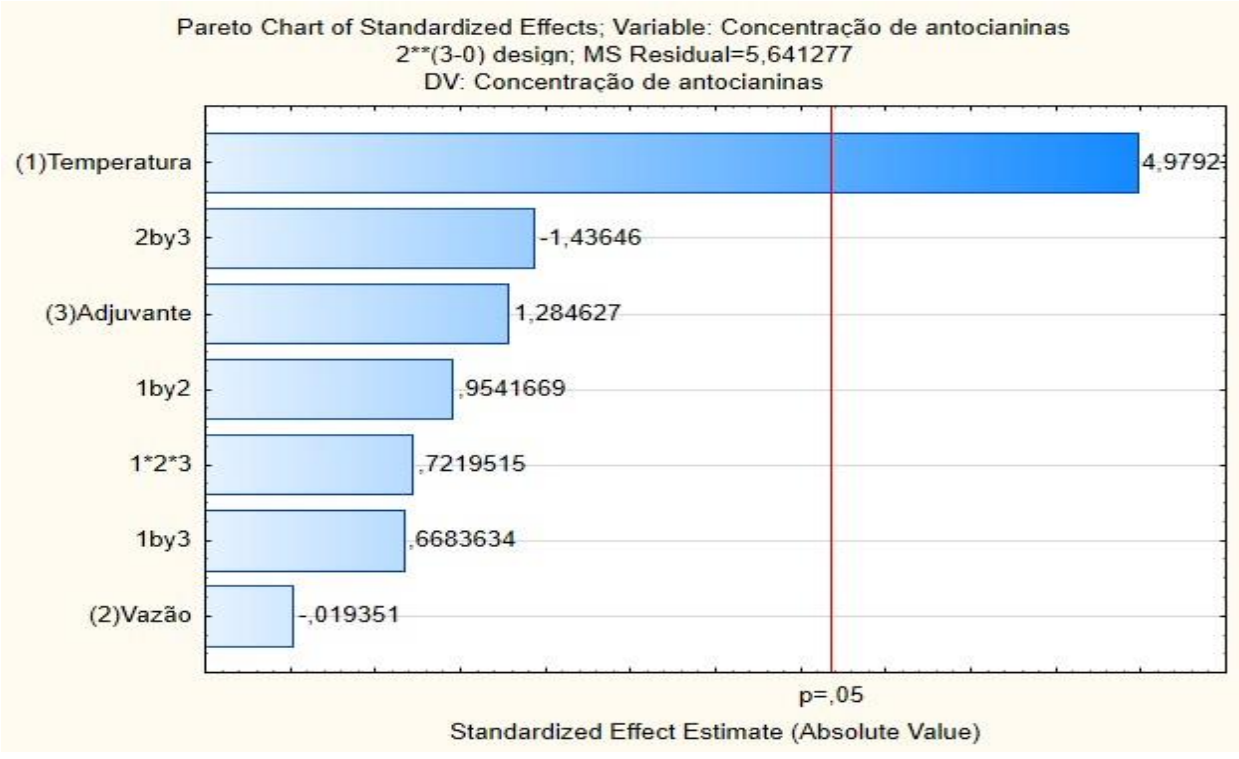

\section{CONCLUSÕES}

Os resultados obtidos neste estudo sugerem que o processo de secagem por atomização utilizando concentrado proteico de soro de leite como adjuvante pode ser uma alternativa para produzir um pigmento natural com propriedades funcionais a partir do resíduo agroindustrial da uva.

\section{AGRADECIMENTOS}

Apoio financeiro da FAPESP 


\section{Congresso Brasileiro de Engenharia Química \\ em Iniciação Científica \\ UFSCar - São Carlos - SP \\ 16 a 19 de Julho de 2017}

QUÍMICA EM INICIACÃO CIENTÍFICA

\section{REFERÊNCIAS}

GALANAKIS, C.M. Separation of functional macromolecules and micromolecules: From ultrafiltration to the border of nanofiltration. Trends in Food Science \& Technology. v. 42, n. 1, p.44-63, 2015.

LAVELLI, V.; SRI HARSHA P.S.C.; PAGLIARINI, E. Degradation kinetics of encapsulated grape skin phenolics and micronized grape skins in various water activity environments and criteria to develop wide-ranging and tailor-made food applications. Innovative Food Science \& Emerging Technologies, v.39, p.156-164, 2017.

LEE, J.; DURST, R. W.; WROLSTAD, R. E. Determination of total monomeric anthocyanin pigment content of fruit juices, beverages, natural colorants, and wines by the $\mathrm{pH}$ differential method: collaborative study. Journal of AOAC international, v. 88, n. 5, p. 1269-1278, 2005.

PÉREZ-JIMÉNEZ et al. Updated methodology to determine antioxidant capacity in plant foods, oils and beverages: Extraction, measurement and expression of results. Food Research International, v. 41, n. 3, p. 274-285, 2008.

SELANI, M.M.; CONTRERAS-CATILLO, C.J.; SHIRAHIGUE, L.D.; GALLO, C.R.; PLATA-OVIEDO M.; MONTES-VILLANUEVA, N. Wine industry residues extracts as natural antioxidants in raw and cooked chicken meat during frozen storage. Meat Science, v. 88, p.397-403, 2011.

SILVA, G.J.F.; CONSTANT, P.B.L.; FIGUEIREDO, R.W.; MOURA, S.M. Formulação e estabilidade de corantes de antocianinas extraídas das cascas de jabuticaba (Myrciaria ssp.). Alimentos e Nutrição, v. 21, n.3, p. 429-436, 2010.

SILVA, M.C.; SOUZA, V.B.; THOMAZINI, M.; SILVA, E.R.; SMANIOTTO, T.; CARVALHO, R.A. et al. Use of the jabuticaba (Myrciaria cauliflora) depulping residue to produce a natural pigment powder with functional properties. LWT - Food Science and Technology, v. 55, p. 203-209, 2014.

TONON, R.V., BRABET, T.C., PALLET, D.; BRAT.P.; \& HUBINGER,M.D. Physicochemical and morphological characterization of acai ( Euterpe oleraceal Mart) powder produced with different carrier agents. International Journal of Food Science \& Technology, v.44, n.10, p.1950-1958, 2009. 